\title{
DIO 3 - TUPLES FOR SPECIAL NUMBERS - I
}

\section{M.A.Gopalan ${ }^{1}$, V.Geetha ${ }^{2}$, S.Vidhyalakshmi ${ }^{3^{*}}$}

1,3. Professors, Department of Mathematics, Shrimathi Indira Gandhi College, Trichy,

2. Assistant Professor, Department of Mathematics, Cauvery College for Women, Trichy.

Keywords: Dio 3 - tuples, Pell equation, Polygonal numbers and Centered polygonal numbers, Linear Polynomials and Jacobasthal-Lucas number.

\begin{abstract}
We search for three distinct polynomials with integer coefficients such that the product of any two members of the set added with their sum and increased by a non- zero integer (or polynomial with integer coefficients) is a perfect square.
\end{abstract}

\section{Introduction:}

The problem of constructing the set with property that the product of any two its distinct elements is one less than a square has a very long history and such sets were studied by Diophantus. A set of $m$ positive integers $\left\{a_{1}, a_{2}, \ldots . a_{m}\right\}$ is called a Diophantine m-tuple if

$$
a_{i} * a_{j}+1 \text { is a perfect square }
$$

a perfect square for all $1 \leq i \prec j \leq m$. Many generalizations of this problem (1) were considered since antiquity, for example by adding a fixed integer $n$ instead of 1 , looking kth powers instead of squares or considering the powers over domains other than $\mathrm{Z}$ or Q. Many mathematicians consider the problem of the existence of Diophantine quadruples with the property $\mathrm{D}(\mathrm{n})$ for any arbitrary integer $\mathrm{n}$ and also for any linear polynomials in $\mathrm{n}$. In this context one may refer [1-16]. The above results motivated us the following definition:

A set of three distinct polynomials with integer coefficient $\left(a_{1}, a_{2}, \mathrm{a}_{3}\right)$ is said to be a special dio 3- tuple with property $\mathrm{D}(\mathrm{n})$ if $a_{i} * a_{j}+\left(a_{i}+a_{j}\right)+n$ is a perfect square for all $1 \leq i \prec j \leq 3$.

In the above definition $n$ may be a non - zero integer or polynomial with integer coefficients. In this communication we consider a few special dio 3 tuples of polygonal numbers from $t_{11, n}$ to $t_{15, n}$, centered polygonal numbers from $c t_{11, n}$ to $c t_{15, n}$, linear polynomials and jacobasthal lucas number with their corresponding properties.

\section{Notations:}

$$
\begin{aligned}
& t_{m, n}=n\left(1+\frac{(n-1)(m-2)}{2}\right)= \\
& c t_{m, n}=\frac{m n(n+1)}{2}+1=\text { Polygonal number of rank n with sides } \mathrm{m} \\
& j_{n}=2^{n}+1=\text { Jacobasthal-Lucas number of rank } \mathrm{n}
\end{aligned}
$$




\section{Construction of Dio 3-tuples for Hendecagonal number:}

Let $a=2 t_{11, n}, \quad b=2 t_{11, n-2}$ be Hendecagonal number of rank $\mathrm{n}$ and $\mathrm{n}-2$ respectively such that $a b+(a+b)+\left(-18 n^{2}+50 n-1\right)$ is a perfect square say $\gamma^{2}$

Let $\mathrm{c}$ be any non zero integer such that

$$
\begin{aligned}
& a c+(a+c)+\left(-18 n^{2}+50 n-1\right)=\alpha^{2} \\
& \mathrm{~b} c+(\mathrm{b}+c)+\left(-18 n^{2}+50 n-1\right)=\beta^{2}
\end{aligned}
$$

On solving equations (2) and (3), we get

$$
(b+1) \alpha^{2}-(a+1) \beta^{2}=(a-b)+\left(-18 n^{2}+50 n-1\right)(b-a)
$$

Assume $\alpha=x+(a+1) T$ and $\beta=x+(b+1) T$ and it reduces to

$$
x^{2}=(b+1)(a+1) T^{2}+\left(-18 n^{2}+50 n-2\right)
$$

The initial solution of equation (5) is given by

$$
T_{o}=1 \text { and } x_{0}=9 n^{2}-25 n+7
$$

Therefore, $\alpha=18 n^{2}-32 n+8$

On substuting the values of $\alpha$ and $a$ in equation (2), we get

$$
\begin{aligned}
c & =36 n^{2}-100 n+65 \\
& =t_{11, n-2}+72 n+135
\end{aligned}
$$

Therefore triple $\left(2 t_{11, n}, 2 t_{11, n-2}, t_{11, n-2}+72 n+135\right)$ is Dio 3- tuple with property $\mathrm{D}\left(-18 n^{2}+50 n-1\right)$

For simplicity, we present below the Dio 3-tuple for polygonal numbers from $t_{11, n}$ to $t_{15, n_{\text {with }}}$ suitable properties.

\begin{tabular}{|c|c|c|c|}
\hline $\mathbf{a}$ & $\mathbf{b}$ & $\mathbf{c}$ & $\mathbf{D}(\mathbf{n})$ \\
\hline \multirow{2}{*}{$2 t_{11, n}$} & $2 t_{11, n-2}$ & $8 t_{11, n}-72 n+67$ & $D(14)$ \\
\cline { 2 - 4 } & $2 t_{11, n-1}$ & $8 t_{11, n-1}+36 n-39$ & $D\left(-9 n^{2}+16 n\right)$ \\
\hline \multirow{3}{*}{$t_{12, n}$} & \multirow{2}{*}{$t_{12, n-2}$} & $t_{12, n}+t_{12, n-1}+2 t_{12, n-2}+12 n-39$ & $D\left(-20 n^{2}+56 n-19\right)$ \\
\cline { 2 - 4 } & $t_{12, n-1}$ & $t_{12, n}+t_{12, n-2}-28 n+11$ & $D(-3)$ \\
\hline \multirow{3}{*}{$2 t_{13, n}$} & \multirow{2}{*}{$2 t_{13, n-2}$} & $2 t_{12, n-1}-6 n-4$ & $D\left(10 n^{3}-27 n^{2}+22 n-5\right)$ \\
\cline { 2 - 4 } & & $8 t_{13, n-2}+88 n-169$ & $D\left(-44 n^{2}+124 n+2\right)$ \\
\hline \multirow{3}{*}{$t_{14, n-1}$} & \multirow{2}{*}{$t_{14, n-2}$} & $2 t_{13,2 n-1}+62 n-63$ & $D(38)$ \\
\cline { 2 - 4 } & \multirow{2}{*}{$t_{14, n-1}$} & $2 t_{13,2 n-2}-26 n+33$ & $D\left(-33 n^{2}+60 n-4\right)$ \\
\hline \multirow{3}{*}{$2 t_{15, n}$} & \multirow{2}{*}{$2 t_{15, n-2}$} & $t_{50, n}-45(n-1)$ & $D\left(28 n^{2}-76 n+74\right)$ \\
\cline { 2 - 4 } & $2 t_{15, n-1}$ & $8 t_{14,2(n-1)}-10 n+13$ & $D(2)$ \\
\cline { 2 - 4 } & & $8 t_{14, n}+2 t_{14, n-1}-4$ & $D\left(-6 n^{2}+11 n-2\right)$ \\
\hline
\end{tabular}




\section{Construction of Dio 3-tuples for Centered Hendecagonal number:}

Let $a=2 c t_{11, n}, b=2 c t_{11, n-2}$ be Centered Hendecagonal number of rank $\mathrm{n}$ and $\mathrm{n}-2$ respectively such that $a b+(a+b)+352 n^{2}-352 n-10$ is a perfect square say $\gamma^{2}$

Let $\mathrm{c}$ be any non zero integer such that

$$
\begin{aligned}
& a c+(a+c)+352 n^{2}-352 n-10=\alpha^{2} \\
& \mathrm{~b} c+(\mathrm{b}+c)+352 n^{2}-352 n-10=\beta^{2}
\end{aligned}
$$

On solving equations (8) and (9), we get

$$
(b+1) \alpha^{2}-(a+1) \beta^{2}=(a-b)+\left(352 n^{2}-352 n-10\right)(b-a)
$$

Assume $\alpha=x+(a+1) T$ and $\beta=x+(b+1) T$, in (10) and it reduces to

$$
x^{2}=(b+1)(a+1) T^{2}+\left(352 n^{2}-352 n-11\right)
$$

The initial solution of equation (11) is given by

$$
T_{o}=1 \text { and } x_{0}=11 n^{2}-11 n+8
$$

Therefore,

$$
\alpha=22 n^{2}+11
$$

On substuting the values of $\alpha$ and $a$ in equation (8), we get

\begin{tabular}{|c|c|c|c|}
\hline $\mathbf{a}$ & b & c & $\mathrm{D}(\mathrm{n})$ \\
\hline \multirow{2}{*}{$2 c t_{11, n}$} & $2 c t_{11, n-2}$ & $c t_{22,2 n-1}-22 n+10$ & $D(-10)$ \\
\hline & $2 c t_{11, n-1}$ & $c t_{88, n}-44 n$ & $D\left(11 n^{2}-4\right)$ \\
\hline \multirow{3}{*}{$c t_{12, n}$} & \multirow{2}{*}{$c t_{12, n-2}$} & $2 c t_{12, n-2}+c t_{24, n}-4$ & $D\left(96 n^{2}-96 n-11\right)$ \\
\hline & & $6 c t_{8, n}-48 n+1$ & $D(-11)$ \\
\hline & $c t_{12, n-1}$ & $4 c t_{12, n}-24 n-1$ & $D\left(12 n^{2}-3\right)$ \\
\hline \multirow{3}{*}{$2 c t_{13, n}$} & \multirow{2}{*}{$2 c t_{13, n-2}$} & $8 c t_{13, n-1}+43$ & $D\left(520 n^{2}-520 n+2\right)$ \\
\hline & & $8 c t_{13, n}-104 n+3$ & $D(14)$ \\
\hline & $2 c t_{13, n-1}$ & $2 c t_{13, n-2}+39 n-29$ & $D\left(13 n^{2}+1\right)$ \\
\hline \multirow{2}{*}{$c t_{14, n}$} & \multirow{2}{*}{$c t_{14, n-2}$} & $c t_{14,2 n-1}-14 n+26$ & $D\left(140 n^{2}-140 n-6\right)$ \\
\hline & & $7 c t_{8, n-1}$ & $D(6)$ \\
\hline
\end{tabular}

$$
\begin{aligned}
c & =44 n^{2}-44 n+43 \\
& =t_{90, n}-n+43
\end{aligned}
$$

Therefore triple $\left(2 c t_{11, n}, 2 \mathrm{c} t_{11, n-2}, t_{90, n}-n+43\right)$ is Dio 3- tuple with property $\mathrm{D}\left(352 n^{2}-352 n-10\right)$

For simplicity, we present below the Diophantine triples for polygonal numbers from $c t_{11, n}$ to $c t_{15, n}$ with suitable properties. 


\begin{tabular}{|c|c|c|c|}
\hline & $c t_{14, n-1}$ & $c t_{14,2 n}-14 n$ & $D\left(7 n^{2}-2\right)$ \\
\hline \multirow{3}{*}{$2 c t_{15, n}$} & \multirow{2}{*}{$2 c t_{15, n-2}$} & $c t_{15,2(n-2)}+270 n-393$ & $D\left(720 n^{2}-720 n+46\right)$ \\
\cline { 3 - 4 } & $2 c t_{15, n-1}$ & $8 c t_{15, n-1}+3$ & $D(46)$ \\
\cline { 3 - 4 } & & $3\left(c t_{40, n-1}+20 n-2\right)$ & $D\left(15 n^{2}+8\right)$ \\
\hline
\end{tabular}

\section{Construction of Dio 3 - tuples for Linear Polynomials}

\section{Case.1:}

Let $a=r-1, b=r-2$ be two linear polynomials such that $a b+a+b+\left(k^{2}+1+(2 k+1) r\right)$ is a perfect square say $\gamma^{2}$

Let $\mathrm{c}$ be any non zero integer such that

$$
\begin{aligned}
& a c+a+c+\left(k^{2}+1+(2 k+1) r\right)=\alpha^{2} \\
& b c+b+c+\left(k^{2}+1+(2 k+1) r\right)=\beta^{2}
\end{aligned}
$$

On solving equation, (14) and (15), we get

$$
(b+1) \alpha^{2}-(a+1) \beta^{2}=(a-b)+\left(k^{2}+1+(2 k+1) r\right)(b-a)
$$

Assuming $\alpha=x+(a+1) T$ and $\beta=x+(b+1) T$ in (16), it reduces to

$$
x^{2}=(b+1)(a+1) T^{2}+\left(k^{2}+1+(2 k+1) r\right)-1
$$

The initial solution of equation (17) is given by

$$
T_{o}=1 \text { and } x_{0}=r+k
$$

Therefore, $\quad \alpha=2 r+k$

On substuting the values of $\alpha$ and $a$ in equation (14), we get

$$
c=4 r+2 k-2
$$

Therefore, triple $(r-1, r-2,4 r+2 k-2)$ is Dio 3-tuple with property $D\left(k^{2}+1+(2 k+1) r\right)$

\section{Case.2:}

Let $a=r+1, b=r+2$ be two linear polynomials such that $a b+a+b+r+4$ is a perfect square say $\gamma^{2}$

Let $\mathrm{c}$ be any non zero integer such that

$$
\begin{aligned}
& a c+a+c+r+4=\alpha^{2} \\
& b c+b+c+r+4=\beta^{2}
\end{aligned}
$$

On solving equation, (20) and (21), we get

$$
(b+1) \alpha^{2}-(a+1) \beta^{2}=(a-b)+(r+4)(b-a)
$$

Assuming $\alpha=x+(a+1) T$ and $\beta=x+(b+1) T$ in (22), it reduces to

$$
x^{2}=(b+1)(a+1) T^{2}+(r+4)-1
$$

The initial solution of equation (17) is given by

$$
T_{o}=1 \text { and } x_{0}=r+3
$$


Therefore, $\quad \alpha=2 r+5$

On substuting the values of $\alpha$ and $a$ in equation (20), we get

$$
c=4 r+10
$$

Therefore, triple $(r+1, r+2,4 r+10)$ is Dio 3-tuple with property $D(r+4)$

\section{Construction of Dio 3 - tuples for Jacobasthal -Lucas number:}

Let $a=j_{2 n}, b=j_{2 n+2}$ be Jacobasthal -Lucas numbers of rank $2 \mathrm{n}$ and $2 \mathrm{n}+2$ respectively, such that $a b+a+b+2.2^{2 n}+6$ is a perfect square say $\gamma^{2}$

Let $\mathrm{c}$ be any non zero integer such that

$$
\begin{aligned}
& a c+a+c+2.2^{2 n}+6=\alpha^{2} \\
& b c+b+c+2.2^{2 n}+6=\beta^{2}
\end{aligned}
$$

On solving equation, (26) and (27), we get

$$
(b+1) \alpha^{2}-(a+1) \beta^{2}=(a-b)+2.2^{2 n}+6(b-a)
$$

Assuming $\alpha=x+(a+1) T$ and $\beta=x+(b+1) T$ in (28), it reduces to

$$
x^{2}=(b+1)(a+1) T^{2}+2.2^{2 n}+5
$$

The initial solution of equation (29) is given by

$$
T_{o}=1 \text { and } x_{0}=2.2^{2 n}+3
$$

Therefore,

$$
\alpha=3.2^{2 n}+5
$$

On substuting the values of $\alpha$ and $a$ in equation (26), we get

$$
\begin{aligned}
& \qquad=9.2^{2 n}+9 \\
& \text { Therefore, triple } \\
& \left(j_{2 n}, j_{2 n+2}, 9 j_{2 n}\right) \\
& \text { is Dio 3- tuple with property } D\left(2.2^{2 n}+6\right)
\end{aligned}
$$

In general, it is noted that the triple $\left(j_{2 n}, j_{2 n+2}, 92^{2 n}+2 k+1\right)$ is a Dio 3-tuple with property $D\left[(4 k-14) 2^{2 n}+k^{2}-2 k-2\right]$

\section{Conclusion:}

In this paper we have presented a few examples of constructing a special Dio 3 tuples for Polygonal numbers, Centered polygonal numbers, linear polynomials and Jacobasthal-lucas numbers with suitable properties. To conclude one may search for Dio 3 - tuples for higher order polygonal numbers and centered polygonal numbers with their corresponding suitable properties.

*The financial support from the UGC, New Delhi (F.MRP-5123/14 (SERO/UGC) dated March 2014) for a part of this work is gratefully acknowledged. 


\section{References:}

[1]. A.F.Beardon and M.N.Deshpande (2007), Diophantine triples, The mathematical Gazette, 86 (2002), 258- 260.

[2]. Bugeaud, A.Dujella and M.Mignotte (2007), On the family of Diophantine triples $\left\{k-1, k+1,16 k^{3}-4 k\right\}$, Glasgow Math.J., 49, 333-344.

[3]. Lj.Bacic, A.Filipin (2013), On the family of $\mathrm{D}(4)-\operatorname{triples}\left\{k-2, k+2,4 k^{3}-4 k\right\}$, Bull. Belg. MTH. Soc. Simon Stevin 20, 777- 787.

[4]. Lj.Bacic, A.Filipin (2013), On the extendibility of D(4)- pairs, Math. Commun. 18, 447 -456.

[5]. M.N.Deshpande (2002), One interesting family of Diophantine triplets, International J.Math.ed. Sci.Tech., 33, 253-256.

[6]. M.N.Deshpande (2003), Families of Diophantine triplets, Bulletin of the Marathwada Mathematical Society, 4, 19-21.

[7]. Y.Fujita (2008), The extensibility of Diophantine pairs $\{k-1, k+1\}$, J.Number theory, 128, 322-353.

[8]. Y.Fujita, A.Togbe (2011), Uniqueness of the extension of the $D\left(4 k^{2}\right){ }^{-}$triple $\left\{k^{2}-4, k^{2}, 4 k^{2}-4\right\}$, Notes Number Theory Discrete Math.17, 42-49.

[9]. M.A.Gopalan and V.Pandichelvi (June 2009), On the extensibility of the Diophantine triple involving Jacobsthal numbers $\left(j_{2 n-1}, j_{2 n+1}-3,2 j_{2 n}+j_{2 n-1}+j_{2 n+1}-3\right)$, International journal of Mathematics \& Application, 2 (1), 1-3.

[10]. M.A.Gopalan and G.Srividhya (2009), Diophantine Qudrapules for Fibonacci numbers with property D(1), Indian Journal of Mathematics and mathematical Sciences, 5 (2), 57-59.

[11]. M.A.Gopalan and G.Srividhya (2010), Diophantine Qudrapules for Pell numbers with property D(1), Antarctica Journal of Mathematics, 7(3), 357-362.

[12]. M.A.Gopalan and V.Pandichelvi (2011), Construction of the Diophantine triple involving Polygonal numbers, Impact J.Sci.Tech., 5(1), 7-11.

[13]. M.A.Gopalan and G.Srividhya (2012), Two special Diophantine Triples, Diophntus J.Math., 1(1), 23-37.

[14]. M.A.Gopalan, V.Sangeetha and Manju Somanath (2014), Construction of the Diophantine polygonal numbers, Sch. J.Eng. Tech. 2, 19 -22.

[15]. M.A.Gopalan, K.Geetha and Manju Somanath (2014), On special Diophantine Triple, Archimedes Journal of Mathematics, 4(1), 37-43.

[16]. L.Szalay, V.Ziegler (2013), On an S-unit variant of Diophantine m-tuples, Publ. Math. Debrecen 83, $97-121$. 